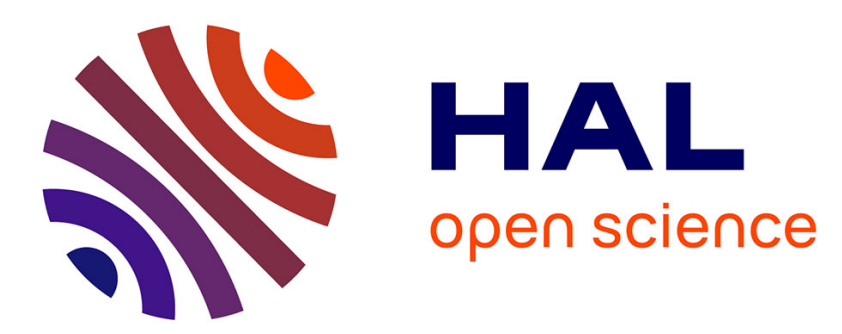

\title{
Nonlinear Phenomena Associated with Large Amplitude Whistler Pulses
}

\author{
R. Stenzel, J. Urrutia, C. Rousculp
}

\section{To cite this version:}

R. Stenzel, J. Urrutia, C. Rousculp. Nonlinear Phenomena Associated with Large Amplitude Whistler Pulses. Journal de Physique IV Proceedings, 1995, 05 (C6), pp.C6-61-C6-66. 10.1051/jp4:1995611 . jpa-00253974

\section{HAL Id: jpa-00253974 https://hal.science/jpa-00253974}

Submitted on 1 Jan 1995

HAL is a multi-disciplinary open access archive for the deposit and dissemination of scientific research documents, whether they are published or not. The documents may come from teaching and research institutions in France or abroad, or from public or private research centers.
L'archive ouverte pluridisciplinaire HAL, est destinée au dépôt et à la diffusion de documents scientifiques de niveau recherche, publiés ou non, émanant des établissements d'enseignement et de recherche français ou étrangers, des laboratoires publics ou privés. 


\title{
Nonlinear Phenomena Associated with Large Amplitude Whistler Pulses
}

\author{
R.L. Stenzel, J.M. Urrutia and C.L. Rousculp
}

Department of Physics, University of California, Los Angeles, CA 90095-154705, U.S.A.

\begin{abstract}
In a magnetized laboratory plasma $\left(n \approx 10^{11} \mathrm{~cm}^{-3}, k T_{e} \geq 1 \mathrm{eV}, B_{0} \geq 10 \mathrm{G}, 1\right.$ $\mathrm{m}$ diam $\times 2.5 \mathrm{~m})$ large amplitude current pulses $(150 \mathrm{~A}, 0.2 \mu \mathrm{s})$ are excited in the parameter regime described by Electron MHD (EMHD; $\omega_{c i} \ll \omega \ll \omega_{c e}$ ). The currents are transported by low-frequency whistlers forming wave packets with topologies resembling 3D spherical vortices. The generalized vorticity, $\boldsymbol{\Omega}=\nabla \times\left(\mathbf{v}+e \mathbf{A} / m_{e}\right)$, is shown to be frozen into the electron fluid, $\partial \boldsymbol{\Omega} / \partial t \approx \nabla \times(\mathbf{v} \times \boldsymbol{\Omega})$. The nonlinearity in $\mathbf{v} \times \boldsymbol{\Omega}$ is negligible since $\mathbf{v}$ and $\boldsymbol{\Omega}(\mathbf{r}, t)$ are found to be nearly parallel. Thus, large amplitude pulses $\left[\Omega(\mathbf{r}, t) \leq \Omega_{0}=e B_{0} / m_{e}\right]$ show the same behavior as small amplitude pulses. However, the associated large currents with $\mathbf{v}_{\text {drift }} \geq\left(k T_{e} / m e\right)^{1 / 2}$ lead to strong electron heating which can modify the damping of whistlers in collisional plasmas $\left(\omega \sim \nu_{e i} \propto T_{e}^{-3 / 2}\right)$. Observations show that a heated flux tube provides a filament of high Spitzer conductivity which permits a nearly collisionless propagation of whistler pulses. This filamentation effect is not associated with density modifications as in modulational instabilities but arises from conductivity modifications. The heated flux tube also generates a quasi-dc magnetic field driven by thermoelectric currents. These assume self-consistently a Taylor state with $\nabla \times \mathbf{B} \approx k \mathbf{B}$.
\end{abstract}

\section{INTRODUCTION}

Time-dependent plasma currents in the Electron MHD regime play an important role in laboratory and space plasmas [1]. Applications include plasma opening switches [2], pulsed ion beam propagation across $\mathbf{B}_{0}$ [3], helicon waves [4], electrodynamic tethers in space [5], dissipation processes in narrow current sheets undergoing reconnection [6], plasma releases [7], and wings behind small asteroids in the solar wind [8]. Basic physics experiments have demonstrated that pulsed EMHD currents in magnetized plasmas are transported by whistler waves [9-11]. An important question is the behavior of large amplitude waves or currents. Observations show that wave packets with energy density exceeding the particle energy density $\left(B^{2} / 2 \mu_{0}>n k T_{e}\right)$ still behave linearly. However, the excitation of such intense pulses leads to strong electron heating. In collisional plasmas, nonuniform rf heating gives rise to nonlinearities, e.g., thermal filamentation [12] and nonuniform damping [13]. Temperature gradients also give rise to thermoelectric currents which last much longer than the transit time of the initial heating pulse. Such long-lasting nonlinear perturbations also play an important role in ionospheric modification experiments [14].

\section{EXPERIMENTAL ARRANGEMENT}

Switched and pulsed currents are generated in a large laboratory plasma schematically shown in Fig. 1. A $1 \mathrm{~m}$ diam $\times 2.5 \mathrm{~m}$ long, magnetized Argon plasma $\left(n \approx 2 \times 10^{11} \mathrm{~cm}^{-3}, k T_{e} \approx 1.4 \mathrm{eV}\right.$, $\left.B_{0} \approx 10 \mathrm{G}, p_{n}=0.3 \mathrm{mTorr}\right)$ is produced with a pulsed $\left(t_{p u l s e} \approx 5 \mathrm{~ms}, t_{\text {rep }} \approx 1 \mathrm{~s}\right)$ dc discharge between an oxide-coated cathode and a gridded anode. In the quiescent, uniform, current-free afterglow plasma $\left(t_{\text {decay }} \approx 20 \mathrm{~ms}\right)$, pulsed currents $\left(I_{\max } \approx 150 \mathrm{~A}, \Delta t \approx 0.2 \mu \mathrm{s}\right)$ are generated with an electrode $(4 \mathrm{~cm}$ diam) biased positively with respect to the end chamber wall. Alterna- 
tively, pulsed currents have also been induced with insulated magnetic loop antennas $(4.5 \mathrm{~cm}$ diam). The time-varying magnetic fields associated with the plasma currents are measured with a triple magnetic probe, recording $\left(B_{x}, B_{y}, B_{z}\right)$, versus time at a given position. By repeating the highly reproducible experiment and moving the probe to $\geq 10,000$ positions in a three-dimensional volume, the vector field $\mathbf{B}(\mathbf{r}, t)$ is obtained with high resolution $(\Delta r \approx 1.5 \mathrm{~cm}, \Delta t=10 \mathrm{~ns})$. Using

Ampère's law, $\mathbf{J}=\nabla \times \mathbf{B} / \mu_{0}$, the current

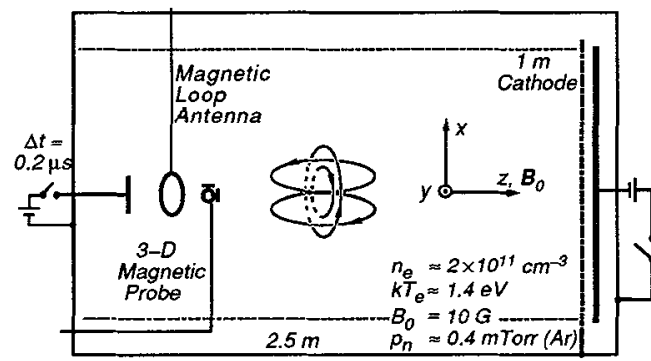

Figure 1. Experimental setup and basic parameters. density is calculated without making any assumptions about field symmetries or $\nabla \cdot \mathbf{B}=0$. All other relevant electrodynamic quantities are obtained via Maxwell's equations and Ohm's law along $\mathbf{B}_{0}$. The differential equations are solved by Fourier transforming the space-time dependence into four-dimensional wavevector-frequency space. The eigenmodes of the transient fields are compared with the dispersion surface of whistlers. The electric field is decomposed into a rotational vector field $(\nabla \times \mathbf{E}=-\partial \mathbf{B} / \partial t)$ and a divergent field $\left(\nabla \cdot \mathbf{E}=\rho / \epsilon_{0}\right)[15]$.

\section{EXPERIMENTAL RESULTS}

When a current pulse is generated by biasing the electrode positive with respect to the chamber wall a magnetic perturbation is generated in the plasma which propagates along $\mathbf{B}_{0}$ at a nearly constant speed. In order to establish the wave nature, the measured time and 3D spatial dependence of $\mathbf{B}(\mathrm{r}, t)$ is Fourier transformed into $\mathrm{k}-\omega$ space. Due to the chosen pulse width, the frequency spectrum is appropriate for EMHD $\left(\omega \approx 0.1 \omega_{c e} \gg \omega_{c i}\right)$. The $\mathbf{k}$-spectrum reflects both the geometry of the exciter and the dispersion of the medium. Due to the symmetry of the axially fed disk electrode, the spectrum of $\mathbf{B}(\mathbf{k})$ depends only on $k_{\perp}$ and $k_{\|}$. For different angles of wave propagation $\left(0<\theta<70^{\circ}\right)$, the distribution of $\mathbf{B}(\mathbf{k}, \omega)$ is found to closely follow the theoretical dispersion of plane, oblique, low frequency whistler waves [16]. Thus, the transient EMHD currents are transported by whistlers analogous to the transport of transient MHD currents by Alfvén waves.

The topology of the field lines within a single-oscillation wavepacket is demonstrated in Fig. 2. Through the measured vector field, $\mathbf{B}(\mathbf{r}, t=0.3 \mu \mathrm{s})$, two sets of field lines are drawn for illustration: the $\left(B_{y}, B_{z}\right)$ lines form a short solenoidal field, while the $\left(B_{x}, B_{y}\right)$ lines form a linked toroidal field in the $x-y$ plane orthogonal to $\mathbf{B}_{0}$. Thus, the wave field has the topology of a $3 \mathrm{D}$ vortex resembling a

Hill's vortex in fluids or a spheromak in fusion plasmas [17]. The current density, $\mathbf{J}$, or electron fluid velocity, $\mathbf{v}=-\mathbf{J} /$ ne, has the same topology since the solenoidal field is generated by a toroidal Hall current and the toroidal field by a linked field-aligned current loop. The net current/field lines are spiralling and their pitch increases with radial distance from the symmetry axis. Special cases include knotted field lines such as trefoils corresponding to the first torus knots. The evolution of the field topology can be explained via Faraday's law and the electron momentum equation. Retaining the electron inertia and introducing the generalized vorticity, $\boldsymbol{\Omega}=\nabla \times \mathbf{v}+e \mathbf{B} / m_{e}$, these two equations can be combined to a single equation describing the evolution of $\Omega$ [18]:

$$
\partial \Omega / \partial t=\nabla \times(\mathbf{v} \times \Omega) .
$$

This equation describes the convection of $\Omega$ in the electron fluid of velocity $\mathbf{v}$. If inertia is neglected, it de-

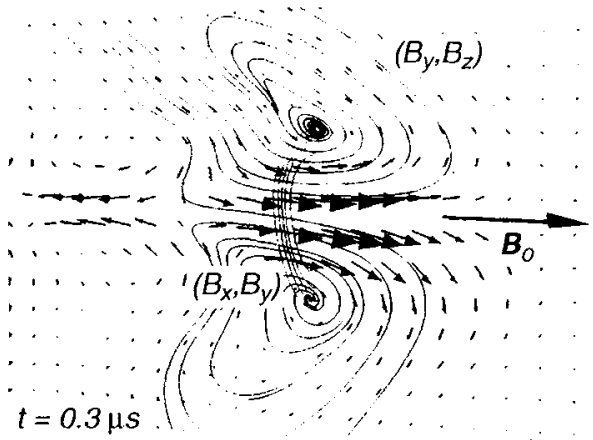

Figure 2. Vector field of the perturbed magnetic field, $\mathbf{B}(\mathbf{r}, t)$, and selected field lines in orthogonal planes. The field topology forms a three-dimensional vortex which propagates in the whistler mode along $\mathbf{B}_{0}$. 
scribes the familiar evolution of magnetic fields frozen into the electrons in EMHD, or frozen into the entire plasma in $\mathrm{MHD}, \partial \mathbf{B} / \partial t=\nabla \times(\mathbf{v} \times \mathbf{B})$.

Since displacement currents are negligible compared to conduction currents, the generalized vorticity in EMHD reduces to $\Omega=\nabla \times \mathbf{v}+e \mathbf{B} / m_{e}=\left(e / m_{e}\right)\left[\left(c^{2} / \omega_{p}^{2}\right) \nabla^{2} \mathbf{B}+\mathbf{B}\right]$. From the measured magnetic field, $\mathbf{B}(\mathbf{r}, t)$, and $\mathbf{B}_{0}$, one readily obtains $\boldsymbol{\Omega}=\boldsymbol{\Omega}(\mathbf{r}, t)+\boldsymbol{\omega}_{c, 0}$, where $\boldsymbol{\omega}_{c, 0}=e \mathbf{B}_{0} / m_{e}$. The transport equation can then be checked experimentally. With $\mathbf{v}=-\mathbf{J} / n e$ and including dissipation, Eq. (1) becomes

$$
\frac{\partial \boldsymbol{\Omega}}{\partial t}=-\frac{\omega_{c, 0}}{n e} \frac{\partial \mathbf{J}}{\partial z}-\frac{1}{n e} \nabla \times[\mathbf{J} \times \boldsymbol{\Omega}(\mathbf{r}, t)]-\frac{\nu}{n e}(\nabla \times \mathbf{J}),
$$

where the convective term has been separated into a linear $(\propto \mathbf{J})$ and a nonlinear $(\alpha \mathbf{J} \times \boldsymbol{\Omega})$ term. The axial components of each term, integrated over the central $x-y$ cross section of positive flux in

$\Omega_{z}$, have been evaluated in time and axial position $z$. Figure 3 displays the peak values as the pulse propagates along $z$ or in time $t^{\prime}=z / v_{\|}$ $\left(v_{\|} \approx 6.7 \times 10^{7} \mathrm{~cm} / \mathrm{s}\right)$. Both the nonlinear convection term and the dissipation term are negligible compared to the linear convection term which accounts, to within measurement accuracy $( \pm 20 \%)$, for the axial flux change. This result demonstrates (i) that the evolution equation for $\boldsymbol{\Omega}$ is satisfied, i.e., $\Omega$ is frozen into the electrons, and (ii), that in spite of large values of $J$ and $\Omega(\mathrm{r}, t)\left(\simeq \Omega_{0}\right)$, the nonlinear term is negligible since $\mathbf{J}$ is nearly parallel to $\Omega$. Furthermore, the weak damping can be accounted for by collisional damping rather than Landau damping (note that $v_{\|} \simeq v_{t h, e}$ ) [19].

Since the fluid nonlinearities $(\mathbf{v} \times \mathbf{B}, \mathbf{v} \cdot \nabla \mathbf{v})$ do not play a significant role, it is possible to generate whistler pulses with large energy densities, $B^{2} / 2 \mu_{0}>n k T_{e, 0}$. However, in such pulses the current density becomes so large that other instabili-

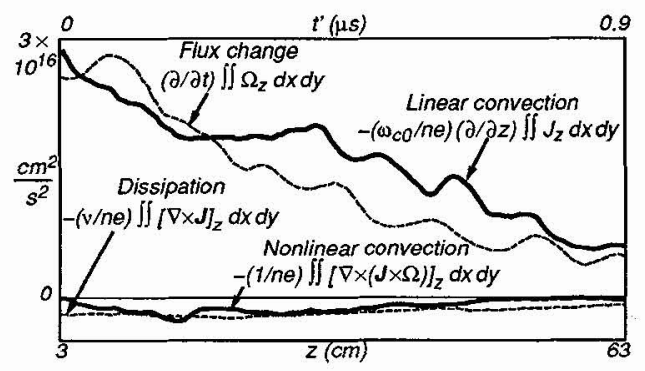

Figure 3. Space-time behavior of the terms describing the evolution of the generalized vorticity, eqn. (2). The axial components are integrated over the transverse cross section for $\Omega_{z}>0$ and their peak values are plotted along the propagation characteristics, $z=v \| t^{\prime}$. The result shows little dissipation and nonlinearity. The generalized vorticity is highly frozen into the electrons and convected linearly for large current pulses ( $I=150 \mathrm{~A}, B_{\max } \approx B_{0}$ ). ties and nonlinearities arise. As an example, Fig. 4 shows contours of current density $J_{z}(x=0, y, z, t=0.2 \mu \mathrm{s})$ for the current pulse described in Fig. 3 $\left(I_{\max }=150 \mathrm{~A}, \Delta t=0.2 \mu \mathrm{s}\right)$. The drift velocity $v_{d}=J_{z} / n e=1.1 \times 10^{8} \mathrm{~cm} / \mathrm{s}$, corresponding to a peak axial current density of $J_{z}=3.4 \mathrm{~A} / \mathrm{cm}^{2}$, exceeds the initial electron thermal velocity $\left(2 k T_{e, 0} / m_{e}\right)^{1 / 2}=7.1 \times 10^{7} \mathrm{~cm} / \mathrm{s}$. Under these conditions, the strong Buneman instability would lead to rapid electron heating [20]. Kelvin-Helmholtz instabilities due to the shear in the electron drift velocity are less important and not observed [6]. Strong electron heating is indeed observed although collisional and transit-time heating may also play a role, in addition to the above-mentioned heating mechanism.

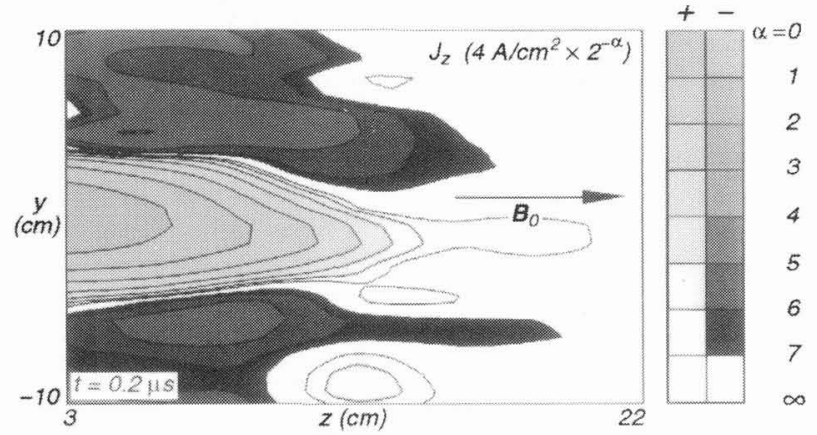

Figure 4. Contours of axial current density, $J_{z}$, at $0.22 \mu$ s after applying a large current pulse $\left(I_{\max }=150 \mathrm{~A}, \Delta t=\right.$ $0.2 \mu \mathrm{s})$ to a disk electrode at $z=0$. The peak electron drift, $v_{d}=J_{z} / n e$, exceeds the initial thermal speed resulting in strong electron heating. The field topology and propagation speed are the same as for small amplitude pulses. 
Modifications of plasma parameters by intense waves/currents can lead to modulational instabilities. While density perturbations are negligible on EMHD time scales, the rapid electron heating in the flux tube of the electrode/antenna forms a filament of high Spitzer conductivity. If the ambient plasma is so collisional that the whistler pulse is normally damped, the high conductivity channel will produce a filamentary radiation pattern. This nonlinear effect is demonstrated in Fig. 5 where contours of axial wave magnetic field $B_{z}(x=0, y, z)$ are displayed at three different times for a $0.2 \mu \mathrm{s}$ long current pulse of small and large amplitudes applied to a $4.5 \mathrm{~cm}$ diam loop antenna. At "small" amplitudes $(I=8 \mathrm{~A})$, the wave damps since the ambient background temperature $\left(k T_{e}=0.5 \mathrm{eV}\right)$ is essentially unchanged by the wave. But at $I=100 \mathrm{~A}$, the electrons in the flux tube of the antenna are rapidly heated $\left(k T_{e} \approx 5 \mathrm{eV}\right)$ and the wave penetrates along the heated channel with little damping. Furthermore, at late times $(t=4.5 \mu \mathrm{s})$, long after the initial heating pulse has traveled through the plasma and dissipated its energy, a nearly constant uniform axial magnetic field remains in the heated flux tube. It is a diamagnetic field driven by $J_{\theta}=-n \nabla_{\perp}\left(k T_{e}\right) \times \mathbf{B}_{0} / B_{0}^{2}$, which satisfies the pressure balance relation $\left(B_{0}-B_{z}\right)^{2} / 2 \mu_{0}+n k \Delta T_{e} \approx B_{0}^{2} / 2 \mu_{0}$, and properly reverses direction with $\mathbf{B}_{0}$. Surprisingly, in addition to the axial diamagnetic field $B_{z}$, a comparatively strong azimuthal field, $B_{\theta}$, is observed.

Figure 5. Nonlinear wave propagation in a collisional plasma due to nonuniform electron heating by the wave. Contours of $B_{z}$ (factor of $2 /$ contour, 7 contours below $B_{\max }=$ $0.75 \mathrm{G}$ ) at different times, $t$, after applying $0.2 \mu$ s current pulses of different magnitudes to a loop antenna at $z=0$. At small amplitude, the fields are strongly damped in space and time $\left(\nu_{e, i} \approx \omega\right)$. At large amplitude, electron heating in the antenna flux tube $\left(k T_{e}=0.5 \mathrm{eV} \rightarrow 5\right.$ $\mathrm{eV}$ ) increases the Spitzer conductivity and permits propagation along the heated plasma filament in the absence of density gradients. Further, a quasi-dc magnetic field remains in the heated flux tube as a result of thermoelectric currents at late times.

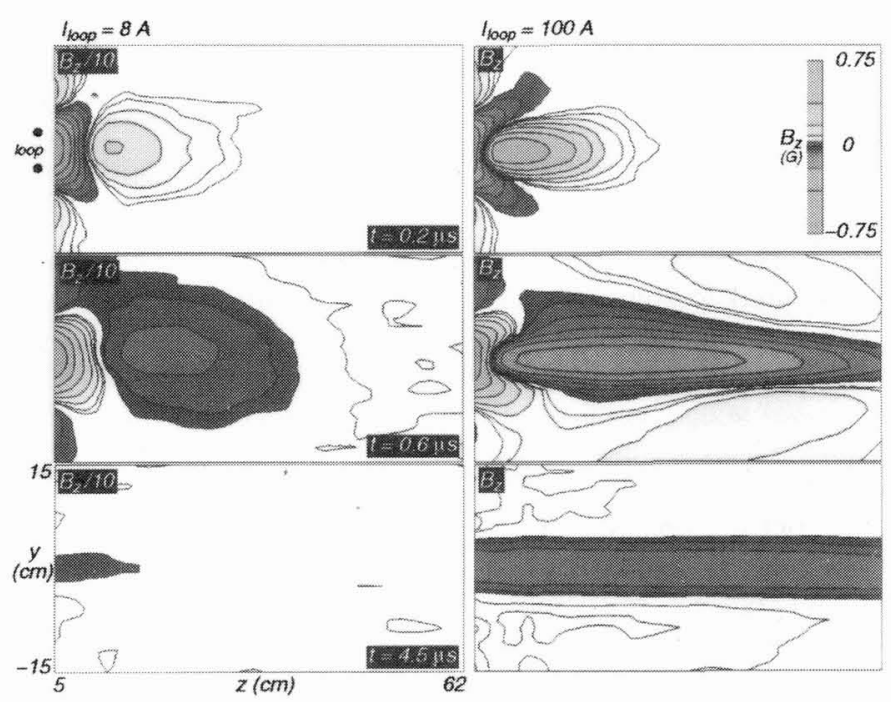

As displayed in Fig. 6, these fields are produced by linked currents, i.e., an azimuthal current $J_{\theta}\left(= \pm J_{x}\right.$ in $x=0$ plane $)$ driven by $\nabla_{\perp}\left(k T_{e}\right)$, and an axial current $J_{z}$ presumably also generated by a weak axial temperature gradient $\left(J_{z}=\alpha \nabla_{\|} k T_{e}\right)$. The axial current is accompanied by a coaxial return current. The variation of $J_{z}$ with $z$ implies the existence of radial currents so that $\nabla \cdot \mathbf{J}=0$. The axial return currents are not induced since $\partial / \partial t=0$ but must arise from space-charge imbalances. The radial component of these space-charge fields produce ion drifts, as discussed by Egorov et al [21]. The radial profiles of the current density or magnetic field are approximately given by the Bessel function solutions to the equation $\nabla \times \mathbf{B}=k \mathbf{B}(\mathbf{r}, t)$, given by $B_{z}=\mu_{0} J_{z}=B_{\text {max }} \mathrm{J}_{0}(k r)$ and $B_{\theta}=\mu_{0} J_{\theta}=B_{\max } \mathrm{J}_{1}(k r)$. Thus, the currents and fields relax to a minimum-energy state as observed and predicted for reverse-field pinches [22]. The currents reverse direction near the edge of the heated flux tube, but decrease in magnitude faster than the Bessel functions since $\left|\nabla k T_{e}\right| \rightarrow 0$. The eigenvalue $k \approx 0.7 \mathrm{~cm}^{-1}$ is either obtained from $k=|\nabla \times \mathbf{B}| / B$ or from the Bessel function fit.

The temporal behavior of the magnetic energy density $B(\mathbf{r}, t)^{2} / 2 \mu_{0}$, current helicity density $\mathbf{J} \cdot \mathbf{B}$, and the angle between $\mathbf{J}$ and $\mathbf{B}, \cos \phi=\mathbf{J} \cdot \mathbf{B} / J B$, are shown in Fig. 7. After the initial pulse has propagated and dissipated $(t>1.5 \mu \mathrm{s})$, the energy in the flux tube is almost constant, but the helicity decays due to a decrease in $|\mathbf{J}| \approx J_{z}$ since $\cos \phi \approx 0.9 \approx$ const. Thus, the diamagnetic current, $J_{\theta}$, decreases slower than the axial current because the radial temperature gradient relaxes 


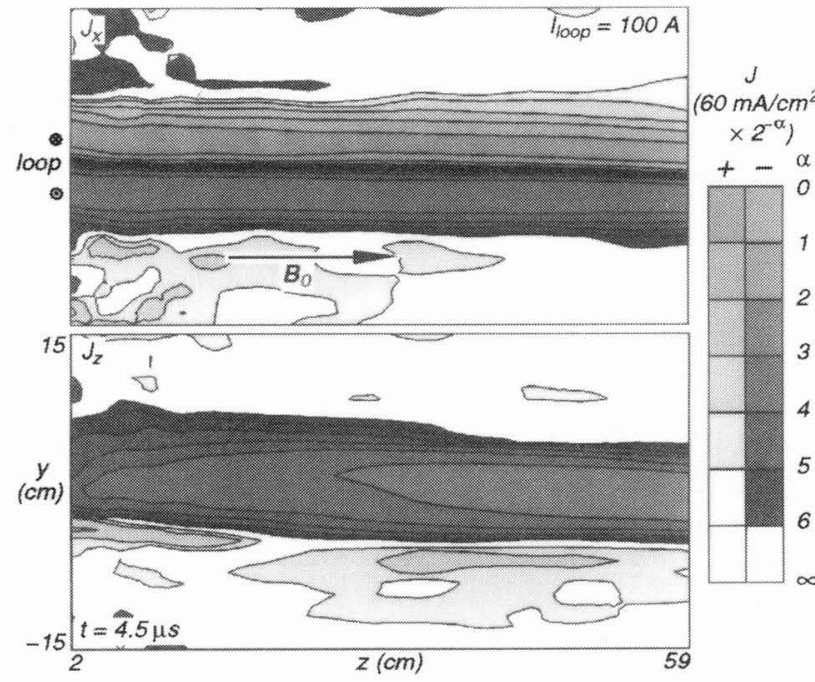

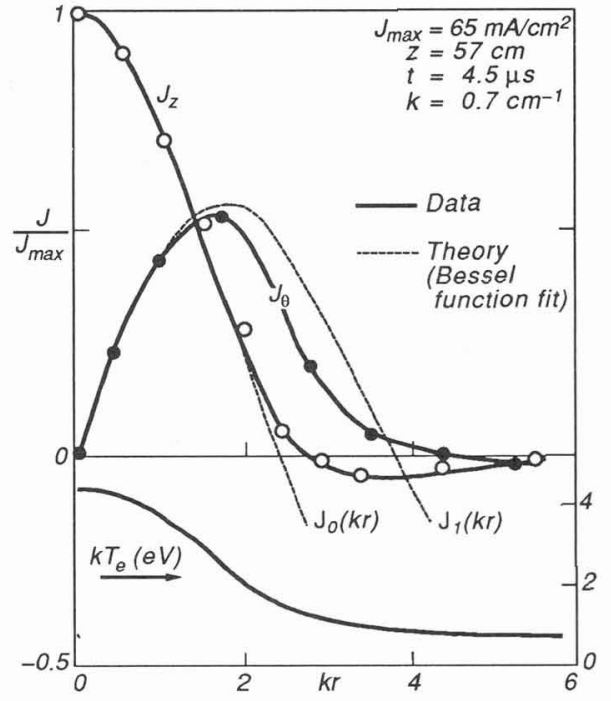

Figure 6. Current distribution in a heated flux tube assuming a minimum energy state. Contours of diamagnetic current density $J_{x}\left(\approx \pm J_{\theta}\right.$ for $\left.x=0\right)$ and field-aligned current, $J_{z}$, at $t=4.5 \mu$ s showing right-handed linkage. The radial current density profiles approximately follow the Bessel function solutions for the differential equation $\nabla \times \mathbf{B} \approx k \mathbf{B}$ with $k \approx 0.7 \mathrm{~cm}^{-1}$, except near the edge of the heated channel where the temperature gradients vanish.

slower than the axial one. Upon reversal of $\mathbf{B}_{0}, J_{z}$ remains unchanged while $B_{z}$ reverses direction, hence the helicity changes sign $(\mathbf{J} \cdot \mathbf{B}<0)$. Although not directly measured, the helicity to either side of the antenna should be equal and of opposite sign. Hence, the total helicity over all space is identically zero. This applies also to the magnetic helicity, $H_{B}=\int \mathbf{A} \cdot \mathbf{B} d V$, if the vector potential, $\mathbf{A}$, is defined in the Coulomb gauge $(\nabla \cdot \mathbf{A}=0)$.

Figure 7. Time dependence of the magnetic energy density $B^{2} / 2 \mu_{0}$, current helicity density $\mathbf{J} \cdot \mathbf{B}$, and the angle $\cos \phi=\mathbf{J} \cdot \mathbf{B} / J B$ in the flux tube $(x \approx y \approx 0, z=30 \mathrm{~cm})$ heated by a large amplitude whistler pulse $(t \leq 1.5 \mu \mathrm{s})$. Due to anisotropic heat flow, the axial current density, $J_{z}$, driven by $\nabla_{\|} k T_{e}$ decays more rapidly than the diamagnetic field $B_{z}$ driven by $\nabla_{\perp} k T_{e}$.

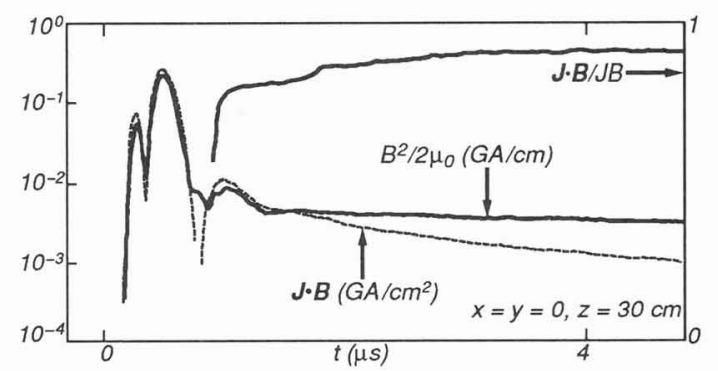

\section{CONCLUSIONS}

Time-varying currents in the EMHD regime propagate in the whistler mode in a uniform, unbounded magnetoplasma. The topology of current pulses is that of $3 \mathrm{D}$ vortices with $\mathbf{J}$ approximately parallel to $\Omega(\mathbf{r}, t)$. The generalized vorticity $\Omega=\nabla \times \mathbf{v}+e \mathbf{B} / m_{e}$ is highly frozen into the electron fluid. Nonlinearities arising from the momentum equation are negligible for $B(r, t) \leq B_{0}$. Strong electron heating occurs when $v>\left(2 k T_{e} / m_{e}\right)^{1 / 2}$, leading to conductivity changes, modulational instabilities in collisional plasmas, and remnant thermoelectric currents. The latter form a quasi-stationary minimum-energy state satisfying $\nabla \times \mathbf{B}=k \mathbf{B}(\mathbf{r}, t)$ in the cylindrical geometry of a heated flux tube. Such nonlinear processes may play a role in ionospheric heating experiments. 


\section{Acknowledgements}

One of the authors (R.L.S.) gratefully acknowledges valuable discussions with Drs. G. Matthieussent, L. Gorbunov, and C. Krafft as well as the support of CNRS during his stay at LPGP in Orsay. We also acknowledge the support of the US National Science Foundation for supporting this basic research project.

\section{References}

[1] Kingsep A.S., Chukbar K.V., and Yan'kov V.V., in Reviews of Plasma Physics, B.B.Kadomtsev, ed. (Consultants Bureau, New York, 1990) 16, 243-291.

[2] Commisso R.J., Goodrich P.J., Grossmann J.M., Hinshelwood D.D., Ottinger P.F., and Weber B.V., Phys. Fluids B 4 (1992) 2368-2376.

[3] Sudan R.N., "Collective Beam Plasma Interactions", in Handbook of Plasma Physics, A.A. Galeev and R.N. Sudan Eds. (North Holland, Amsterdam,1984) II, p.337.

[4] Light M. and Chen F.F., Phys. Plasmas 2 (1994) 1084-1093.

[5] Dobrowolny M. and Melchioni E., J. Geophys. Res. 98 (1993) 13761-13778.

[6] Drake J.F., Kleva R.G., and Mandt M.E., Phys. Rev. Lett. 73 (1994) 1251-1254.

[7] Haerendel G., Paschmann G., Baumjohann W., and Carlson C.W., Nature 320 (1986) 720-723.

[8] Kivelson M.G., Bargatze L. F., Khurana K. K., Southwood D. J., Walker R. J., and Coleman P. J., Jr., Science (1993) 261, 331-334.

[9] Urrutia J.M. and Stenzel R.L., Phys. Rev. Lett. 62 (1989) 272-275.

[10] Stenzel R.L. and Urrutia J.M., J. Geophys. Res. 95 (1990) 6209-6226.

[11] Stenzel R.L., Urrutia J.M., and Rousculp C.L., Phys. Plasmas 2 (1995) 1114-1128.

[12] Stenzel R.L., J. de Physique C6 (1977) 89-102.

[13] Urrutia J.M. and Stenzel R.L., Phys. Rev. Lett. 67 (1991) 1867-1870.

[14] Keskinen M.J., Chaturvedi P.K., and Ossakow S.L., Radio Sci. 28 (1993) 775-784.

[15] Rousculp C.L., Stenzel R.L., and Urrutia J.M., Phys. Rev. Lett. 72 (1994) 1658-1661.

[16] Helliwell R.A., Whistlers and Related Ionospheric Phenomena (Stanford Univ. Press, Stanford, CA, 1965) pp. 23-24.

[17] Dolan T.J., Fusion Research (Pergamon, New York, 1982) II, pp. 329-335.

[18] Yan'kov V.V., Sov. Phys. JETP 80 (1995) 219-227.

[19] Chen F.F., Plasma Phys. Contr. Fusion 33 (1991) 339-364.

[20] Buneman O., Phys. Rev. 115 (1959) 503-517.

[21] Egorov S.V., Kostrov A.V., and Tronin A.V., JETP Lett. 47 (1988) 102-106.

[22] Taylor J.B., Rev. Mod. Phys. 58 (1986) 741. 